
\title{
28 Research Suare \\ Primary Low Grade-Extrauterine Endometrial Stromal Sarcoma: Analysis of 10 Cases
}

\section{You Wu}

Department of Gynecology, Chinese Academy of Medical Sciences Cancer Institute and Hospital, Beijing Nan Li ( $\triangle$ concerto2000@126.com )

Department of Gynecology, Chinese Academy of Medical Sciences Cancer Institute and Hospital, Beijing Rong Zhang

Department of Gynecology, Chinese Academy of Medical Sciences Cancer Institute and Hospital, Beijing Ping Bai

Department of Gynecology, Chinese Academy of Medical Sciences Cancer Institute and Hospital, Beijing

\section{Research Article}

Keywords: extra-uterine endometrial stromal sarcoma, clinicopathological features, complete resection, oral hormonal therapy, recurrence rate

Posted Date: June 7th, 2021

DOl: https://doi.org/10.21203/rs.3.rs-521228/v1

License: (c) (i) This work is licensed under a Creative Commons Attribution 4.0 International License. Read Full License 


\section{Abstract \\ Background}

This study aimed to analyze the clinical and pathological features of extrauterine endometrial stromal sarcoma (EESS) and explore an effective therapeutic regimen to reduce the recurrence rate in low-grade EESS patients.

\section{Methods}

Ten LG-EESS patients who were treated at the Chinese Academy of Medical Sciences Cancer Institute and Hospital from June 1999 to June 2019 were collected and analyzed.

\section{Results}

(1) Patient demographics is summarized in Table 1. Preoperative CA125 examination showed that 8 patients had a median level of $49.5 \mathrm{U} / \mathrm{L}$ (15.4-168.0 U/L). (2)All ten patients underwent tumor cytoreductive surgery. Five patients underwent optimal tumor resection, achieved a R0 resection. After the initial surgery ,two patients were treated with adjuvant chemotherapy only, two patients were treated with chemotherapy and radiation therapy, and three patients were treated with chemotherapy plus hormonal treatment.(2) Most EESS patients had multiple tumors. the omentum was the most commonly affected site, followed by ovaries.(3) The median follow-up was 94 (range: 27-228) months, and recurrence was observed in 3 patients $(n=10,30 \%)$. The 5-year and 10-year DFS rates were both $70 \%$, as shown in Fig. 1 . OS was both $100 \%$ at 5 and 10 years.

\section{Conclusion}

As a conclusion, EESS is a rare disease and LG-EESS has a good prognosis. Surgery remains the available treatment for patients. LG-EESS has a risk of late recurrence which requires a long-term followup. With a limited sample size, our study shows optimal tumor reductive surgery and adjuvant hormone therapy can significantly reduce the risk of recurrence.

\section{Background}

Endometrial stromal sarcoma (ESS) is a malignant stromal cell tumor that originates from endometrial stromal cells, and it accounts for approximately $1 \%$ of uterine malignancies and less than $10 \%$ of uterine stromal tumors [1]. ESS usually originates in the uterus, but extra-uterine ESS (EESS), which does not involve the uterus, is also found in clinical practice. Currently, there are two theories that explain the origin of EESS. One theory is that EESS originates from the malignant transformation of endometriosis, and the other theory is that it originates from the malignant transformation of Muller cells, residual cells from 
embryogenesis that are widely distributed in the peritoneal and pelvic cavities [2,3]. Malignant endometriosis lesions are mostly endometrioid adenocarcinoma or clear cell carcinoma, and EESS is extremely rare [4].

By searching PubMed for literature published between 1970 and 2016 regarding EESS, Alcazar et al. [5] found that among 76 cases of EESS that originated from endometriosis, the most commonly involved sites were ovary $(44.3 \%)$, pelvic organs outside the uterus and ovaries $(15.2 \%)$, sigmoid rectum $(7.6 \%)$ and small intestine (7.6\%). Masand et al. [6] also found that the ovaries, small intestine, peritoneum, pelvic cavity and vagina were the most commonly affected sites. Currently, the clinical treatment for EESS is still controversial. The treatment for two classes of EESS, low-grade ESS (LG-EESS) and highgrade ESS (HG-EESS) are different, which the former class has a preferable prognosis. Besides intervention of adjuvant therapy, tumor stage and myometrial invasion all contributed to the relapse free survival [7]. The reported overall disease-specific 5-year and 10 -year survival rates are $80-90 \%$ and $70 \%$, respectively, with a high recurrence risk $[8,9]$.

Here we report the study of ten pathologic confirmed LG-EESS patients accepted adjuvant therapy with a follow-up of 27-228 months aiming to determine possible prognosis factors and suggest treatments to potentially reduce EESS relapse.

\section{Method}

\section{Patients}

The present study included patients with histologically proven low-grade EESS who were admitted and treated at the Chinese Academy of Medical Sciences Cancer Institute and Hospital between June 1999 and June 2019. Thirteen patients were included initially, and the clinical and pathological data were retrospectively reviewed. Ten patients were confirmed with no uterine lesions by surgery and postoperative pathology or preoperative imaging examination (pelvic MR and PET-CT), three patients with a history of uterine surgery were excluded due to difficulties to confirm uterine lesions. Patient information was obtained by searching medical records or telephone calls and questionnaires reach to primary care physicians and/or attending oncologists: age at the time of diagnosis, presenting symptoms and signs, previous history of hysterectomy (if applicable), surgical procedures, pathologic features, and recurrence and survival follow-up information. Institutional review board approval was obtained prior to the initiation of this study.

\section{Treatment protocol}

All patients received tumor cytoreductive surgery including resection of both uterine appendages, the omentum, part of the intestinal tube, abdominal and pelvic lymph nodes, or the affected vaginal vulva according their clinicopathological characteristics. Adjuvant treatment was personalized based on the extent of the disease, medical comorbidities, and physician's recommendation. Adjuvant treatments including chemotherapy, radiotherapy, and hormonal treatment, in combination or alone after per- 
treatment evaluation. The chemotherapy regimens were PEI (cisplatin + epirubicin + ifosfamide), TC (paclitaxel + carboplatin) and gemcitabine plus docetaxel. Adjuvant radiotherapy was conducted through . Hormone treatment included megestrol (Megace, progesterone derivative), letrozole (Femara, nonsteroidal aromatase inhibitor) and tamoxifen, which were used mono or combined.

\section{Follow-up}

After the completion of the initial treatment, the follow-up was conducted with the treatment in patients. Relapse was defined as the occurrence of new measurable lesion with clinical or imaging evidence and pathologically confirmed. Disease-free survival (DFS) was defined in months as the time from the date of initial surgery to the date of disease relapse. Overall survival (OS) was calculated in months as the time from the date of initial surgery to the date of death from the disease.

\section{Statistical analysis}

All statistical analyses were performed using SPSS 23.0 (IBM Corp, Armonk, NY). Data of the current study were presented using the mean, standard deviation, median, range, ratio, and/or frequency. The Kaplan-Meier method was used to generate survival curves and rates.

\section{Results}

\section{Patient characteristics}

Patient demographics is summarized in Table 1. The median age of the patients at initial diagnosis was 44.5 years (range: 33 to 66 years). Clinical manifestations mainly included lower abdominal discomfort or pain $(5 / 10,50 \%)$, vaginal bleeding $(2 / 10,20 \%)$, dysmenorrhea $(1 / 10,10 \%)$ and pruritus vulvae $(1 / 10$, $10 \%)$, one patient had no obvious clinical symptoms and was diagnosed during routine physical examination. Four patients had a history of uterine surgery, and pathology results indicated that they all had benign uterine lesions (uterine fibroids or adenomyosis). Preoperative CA125 examination showed that 8 patients had a median level of $49.5 \mathrm{U} / \mathrm{L}$ with range from 15.4-168.0 U/L, of whom 5 had elevated CA125 levels. 
Table 1

Clinical profile of the 10 patients with LG-EESS

\begin{tabular}{|c|c|c|}
\hline Parameter & Number & Percent (\%) \\
\hline Age at diagnosis (median, range), year & 44.5 & $33-66$ \\
\hline \multicolumn{3}{|l|}{ Initial clinical presentation } \\
\hline Vaginal bleeding & 2 & 20 \\
\hline Abd distention & 3 & 30 \\
\hline Abd pain & 2 & 20 \\
\hline Pruritus vulvae & 1 & 10 \\
\hline Dysmenor-rhea & 1 & 10 \\
\hline None & 1 & 10 \\
\hline \multicolumn{3}{|l|}{ History of gynecologic surgery } \\
\hline TAH for leiomyoma & 4 & $40 \%$ \\
\hline CA125 (median, range), U/ml & 49.5 & $15.4-168.0$ \\
\hline \multicolumn{3}{|l|}{ Site of tumor } \\
\hline Vaginal wall & 2 & 20 \\
\hline Sigmoid & 3 & 30 \\
\hline Right ovary & 3 & 30 \\
\hline Vulva & 1 & 10 \\
\hline Pelvis & 1 & 10 \\
\hline \multicolumn{3}{|l|}{ Initial surgery } \\
\hline Optimal tumor resection & 5 & $50 \%$ \\
\hline \multicolumn{3}{|l|}{ Adjuvant treatment } \\
\hline QT & 2 & 20 \\
\hline HT & 3 & 30 \\
\hline $\mathrm{QT}+\mathrm{RT}$ & 2 & 20 \\
\hline $\mathrm{QT}+\mathrm{HT}$ & 3 & 30 \\
\hline Follow up (median, range), month & 94 & $27-228$ \\
\hline
\end{tabular}

$\mathrm{RT}$ = radiotherapy, $\mathrm{QT}$ = chemotherapy, $\mathrm{HT}$ = hormone therapy, $\mathrm{AWD}=$ alive with disease, $\mathrm{NED}=$ no evidence of disease 


\begin{tabular}{|lll|}
\hline Parameter & Number & Percent (\%) \\
\hline Relapse & 3 & 30 \\
\hline Current status & & 30 \\
\hline AWD & 3 & 70 \\
\hline NED & 7 & \\
\hline $\begin{array}{l}\text { RT = radiotherapy, QT = chemotherapy, HT = hormone therapy, AWD = alive with disease, NED = no } \\
\text { evidence of disease }\end{array}$ & \\
\hline
\end{tabular}

According to surgical records and pathological reports, the tumors lesion in all cases located outside the uterus, with diameters ranging from $5 \mathrm{~cm}$ to $20 \mathrm{~cm}$, and most patients had multiple tumors $(8 / 10,80 \%)$ with varying degrees of adhesion to the surrounding tissues or organs. The tumors were lobulated with capsule formation. Some tumors appeared grayish white with a slightly tough texture, and some appeared grayish pink or grayish brown with a soft texture resembled fish meat. Patients with large tumors had hemorrhagic necrosis and occasional focal cystic changes. Among the eight EESS patients bearing multiple tumors, the omentum was the most commonly affected site, followed by ovaries.

For the morphology of low-grade EESS, most cells were small and round or in a short fusiform shape, which most were atypical. The mitotic index was $<10 / 10 \mathrm{HPF}$, and the interstitium was rich in small arterial blood vessels around tumor cells which aligned spirally. As shown in Table 2, among the 10 patients, 2 had localized smooth muscle differentiation, and 7 had definite endometriosis. In addition, 6 were estrogen receptor (ER) positive, 6 were progesterone receptor (PR) positive, and 7 were CD10 positive. Desmin was analyzed in 5 patients with $4(80 \%)$ was negative. Smooth muscle antigen (SMA) were analyzed in 6 patients and 4 (66.7\%) were negative. 
Table 2

\begin{tabular}{|c|c|c|}
\hline Parameter & Number & Percent (\%) \\
\hline Tumor diameter (median, range), cm & 8.7 & $5-20$ \\
\hline Localized smooth muscle differentiation & 2 & 20 \\
\hline Definite endometriosis & 7 & 70 \\
\hline \multicolumn{3}{|l|}{ ER } \\
\hline positive & 5 & 50 \\
\hline \multicolumn{3}{|l|}{ PR } \\
\hline positive & 5 & 50 \\
\hline \multicolumn{3}{|l|}{ CD 10} \\
\hline positive & 7 & 70 \\
\hline \multicolumn{3}{|l|}{ Desmin $(n=5)$} \\
\hline positve & 1 & 20 \\
\hline \multicolumn{3}{|l|}{ SMA $(n=6)$} \\
\hline positive & 2 & 33.3 \\
\hline
\end{tabular}

\section{Surgical And Adjuvant Treatment}

All ten patients underwent tumor cytoreductive surgery including resection of both uterine appendages, the omentum, part of the intestinal tube, abdominal and pelvic lymph nodes and the affected vaginal vulva. Five patients underwent optimal tumor resection, with no residual tumor seen by the naked eye and achieved a R0 resection.

Two patients were treated with adjuvant chemotherapy only after the initial surgery, two patients were treated with chemotherapy and radiation therapy, and three patients were treated with chemotherapy plus hormonal treatment. The average chemotherapy treatment duration was 6 month, 5.2 courses (range: 46). Three patients received only adjuvant hormonal treatment utilizing a variety of agents, the duration of medication used varied from 3 months to 1 year.

\section{Follow-up}


The median follow-up was 94 (range: $27-228)$ months, and recurrence was observed in 3 patients $(\mathrm{n}=10$, $30 \%$ ). The disease-free interval was 25,36 , and 60 months, respectively. The recurrences were all developed abdominopelvic, and one patient had ureteral involvement. Two of the relapsed patients received adjuvant chemotherapy combined radiation therapy, one received chemotherapy only. No satisfactory cytoreductive surgery performed for relapsed patients. The 5-year and 10-year DFS rates were both $70 \%$, as shown in Figure 1. No death due disease progression in all patients. As a result, OS was both $100 \%$ at 5 and 10 years.

\section{Discussion}

The symptom of EESS usually involve abnormal uterine bleeding, however, an accurate diagnosis is more challenging [10]. For patients in our study, three showed abdominal distention, two with vaginal bleeding and two with abdominal pain for initial clinical appearance. To assist diagnosis, preoperative curettage and pathology, including immunohistochemistry test, are import methods [11]. LG-EESS characteristically showed positive for CD10, ER and PR [7, 12].

In our study, small endometriosis lesions in both vaginal and vulva ESS were found which contradict with previous research $[8,9,13]$. Although there might be issues for specimen collection, a possibility that vaginal or vulva EESS involved malignant transformation of endometrial stromal cells still existing.

The treatment option for LG-EESS involved a total abdominal hysterectomy, however, ovary removal and comprehensive surgical staging, including pelvic and para-aortic lymphadenectomy remained debatable [14]. In our study, patient received radical surgery, which was uterine and double appendage resection plus tumor resection, achieved optimal tumor reduction with a lower tumor recurrence rate.

Four patients underwent pelvic lymphadenectomy, a postoperative pathology showed negative in lymph nodes. This also supported the findings that ESS usually did not develop lymphatic metastasis [10]. We also did not directly observe clinical benefit of lymphadenectomy which an analysis of EESS patient showed addition of lymphadenectomy to hysterectomy did not improve either cause-specific survival or overall survival compared to hysterectomy alone $[15,16]$.

For EESS patients of reproductive age, double appendage removal is also recommended [17]. However, a recent study suggested that ovary preservation did not significantly affect the overall survival of patients $[15,18]$. In our study, one young patient with LG-EESS underwent fertility preservation surgery (preservation of the normal uterus and appendages), and adjuvant treatment including chemotherapy and hormone therapy. The patient did not relapse in the follow-up with a NED (no evidence of disease) reached 86 months. Previous studies showed two patients with vaginal EESS after local tumor resection with negative margins did not relapse in 36-months and 38-months follow-ups [8, 13]. These studies indicate that fertility preservation surgery can be performed with cautions in EESS patients follow a desire to retain reproductive ability, however, this conclusion need to be confirmed with large-sample-size studies. 
Postoperative adjuvant treatment of EESS includes radiotherapy, chemotherapy and hormone therapy [19-22]. Our study showed postoperative radiotherapy and chemotherapy did not reduce the tumor recurrence rate but hormone therapy, especially for patients who underwent optimal tumor reduction surgery, had significantly reduced recurrence rate compared to that of patients who did not undergo hormone therapy. As a hormone receptor positive tumor, study showed that LG-EESS was sensitive for endocrine treatment [14].

Patients with LG-EESS had a good prognosis and long-term survival but a risk of late stage recurrence [23]. In our study, the recurrence rate was $30 \%$ and the longest recurrence duration was 60 months.

\section{Conclusion}

As a conclusion, EESS is a rare disease and LG-EESS has a good prognosis. Surgery remains the available treatment for patients. LG-EESS has a risk of late recurrence which requires a long-term followup. With a limited sample size, our study shows optimal tumor reductive surgery and adjuvant hormone therapy can significantly reduce the risk of recurrence.

\section{List Of Abbreviations}

Endometrial stromal sarcoma (ESS)

Low-grade ESS (LG-EESS)

High-grade ESS (HG-EESS)

Disease-free survival (DFS)

Overall survival (OS)

Estrogen receptor (ER)

Smooth muscle antigen (SMA)

No evidence of disease (NED)

\section{Declarations}

\section{Ethics approval and consent to participate}

This study was approved by the ethic committee of Chinese Academy of Medical Sciences Cancer Institute and Hospital. All procedures performed in studies involving human participants were in accordance with the ethical standards of the institutional and/or national research committee and with the 1964 Helsinki declaration and its later amendments or comparable ethical standards. Written informed consent was obtained from the patients. 
Consent for publication

Written informed consent was obtained from the patients.

Availability of data and materials

All data generated or analysed during this study are included in this published article.

\section{Competing interests}

The authors declare that they have no competing interests.

\section{Funding}

None

\section{Authors' contributions}

YW and NL carried out the studies, participated in collecting data, and drafted the manuscript. YW and NL performed the statistical analysis and participated in its design. RZ and PB helped to draft the manuscript. All authors read and approved the final manuscript.

\section{Acknowledgements}

None

\section{References}

1. Ali RH, Rouzbahman M: Endometrial stromal tumours revisited: an update based on the $\mathbf{2 0 1 4}$ WHO classification. Journal of clinical pathology 2015, 68(5):325-332.

2. Chang KL, Crabtree GS, Lim-Tan SK, Kempson RL, Hendrickson MR: Primary extrauterine endometrial stromal neoplasms: a clinicopathologic study of $\mathbf{2 0}$ cases and a review of the literature. International journal of gynecological pathology : official journal of the International Society of Gynecological Pathologists 1993, 12(4):282-296.

3. Jung SI, Shin SS, Choi C, Hwang EC, Kim SO, Kang TW: Endometrial stromal sarcoma presenting as prevesical mass mimicking urachal tumor. Journal of Korean medical science 2009, 24(3):529-531.

4. Khan AW, Craig M, Jarmulowicz M, Davidson BR: Liver tumours due to endometriosis and endometrial stromal sarcoma. HPB : the official journal of the International Hepato Pancreato Biliary Association 2002, 4(1):43-45.

5. Alcázar JL, Guerriero S, Ajossa S, Parodo G, Piras B, Peiretti M, Jurado M, Idoate M: Extragenital endometrial stromal sarcoma arising in endometriosis. Gynecologic and obstetric investigation 2012, 73(4):265-271. 
6. Masand RP, Euscher ED, Deavers MT, Malpica A: Endometrioid stromal sarcoma: a clinicopathologic study of 63 cases. The American journal of surgical pathology 2013, 37(11):1635-1647.

7. Corpa MV, Serafini EP, Bacchi CE: Low-grade endometrial stromal sarcoma presenting as vaginal nodule. Annals of diagnostic pathology 2004, 8(5):295-298.

8. Prat J, Mbatani: Uterine sarcomas. International journal of gynaecology and obstetrics: the official organ of the International Federation of Gynaecology and Obstetrics 2015, 131 Suppl 2:S105-110.

9. Amant F, Moerman P, Cadron I, Neven P, Berteloot P, Vergote I: The diagnostic problem of endometrial stromal sarcoma: report on six cases. Gynecologic oncology 2003, 90(1):37-43.

10. Cui R, Yuan F, Wang Y, Li X, Zhang Z, Bai H: Clinicopathological characteristics and treatment strategies for patients with low-grade endometrial stromal sarcoma. Medicine 2017, 96(15):e6584.

11. Hwang H, Matsuo K, Duncan K, Pakzamir E, Pham HQ, Correa A, Fedenko A, Mhawech-Fauceglia P: Immunohistochemical panel to differentiate endometrial stromal sarcoma, uterine leiomyosarcoma and leiomyoma: something old and something new. Journal of clinical pathology 2015, 68(9):710717.

12. Ronen S, Narula N, Koizumi JH, Hunt B, Giorgadze T: Low-grade endometrial stromal sarcoma presenting as multiple pulmonary nodules: A potential pitfall in fine needle aspiration and core biopsy specimens - A Cytological - Pathological Correlation. Annals of diagnostic pathology 2018, 36:38-43.

13. Oliva E, Garcia-Miralles N, Vu Q, Young RH: CD10 expression in pure stromal and sex cord-stromal tumors of the ovary: an immunohistochemical analysis of 101 cases. International journal of gynecological pathology : official journal of the International Society of Gynecological Pathologists 2007, 26(4):359-367.

14. Mizuno M, Yatabe $Y$, Nawa A, Nakanishi T: Long-term medroxyprogesterone acetate therapy for lowgrade endometrial stromal sarcoma. International journal of clinical oncology 2012, 17(4):348-354.

15. Shah JP, Bryant CS, Kumar S, Ali-Fehmi R, Malone JM, Jr., Morris RT: Lymphadenectomy and ovarian preservation in low-grade endometrial stromal sarcoma. Obstetrics and gynecology 2008, 112(5):1102-1108.

16. Chan JK, Kawar NM, Shin JY, Osann K, Chen LM, Powell CB, Kapp DS: Endometrial stromal sarcoma: a population-based analysis. British journal of cancer 2008, 99(8):1210-1215.

17. Sciallis AP, Bedroske PP, Schoolmeester JK, Sukov WR, Keeney GL, Hodge JC, Bell DA: High-grade endometrial stromal sarcomas: a clinicopathologic study of a group of tumors with heterogenous morphologic and genetic features. The American journal of surgical pathology 2014, 38(9):11611172.

18. Li AJ, Giuntoli RL, 2nd, Drake R, Byun SY, Rojas F, Barbuto D, Klipfel N, Edmonds P, Miller DS, Karlan BY: Ovarian preservation in stage I low-grade endometrial stromal sarcomas. Obstetrics and gynecology 2005, 106(6):1304-1308.

19. Oliva E, Egger JF, Young RH: Primary endometrioid stromal sarcoma of the ovary: a clinicopathologic study of 27 cases with morphologic and behavioral features similar to those of uterine low-grade 
endometrial stromal sarcoma. The American journal of surgical pathology 2014, 38(3):305-315.

20. Xie W, Bi X, Cao D, Yang J, Shen K, You Y: Primary endometrioid stromal sarcomas of the ovary: a clinicopathological study of 14 cases with a review of the literature. Oncotarget 2017, 8(38):6334563352.

21. Leath CA, 3rd, Huh WK, Hyde J, Jr., Cohn DE, Resnick KE, Taylor NP, Powell MA, Mutch DG, Bradley WH, Geller MA et al: A multi-institutional review of outcomes of endometrial stromal sarcoma. Gynecologic oncology 2007, 105(3):630-634.

22. Barney B, Tward JD, Skidmore T, Gaffney DK: Does radiotherapy or lymphadenectomy improve survival in endometrial stromal sarcoma? International journal of gynecological cancer : official journal of the International Gynecological Cancer Society 2009, 19(7):1232-1238.

23. Liu Z, Ding J, Li X, Yu K: Endometrial stromal sarcoma arising in vagina.International Journal of Clinical and Experimental Pathology 2013, 6(12):2997-3002.

\section{Figures}




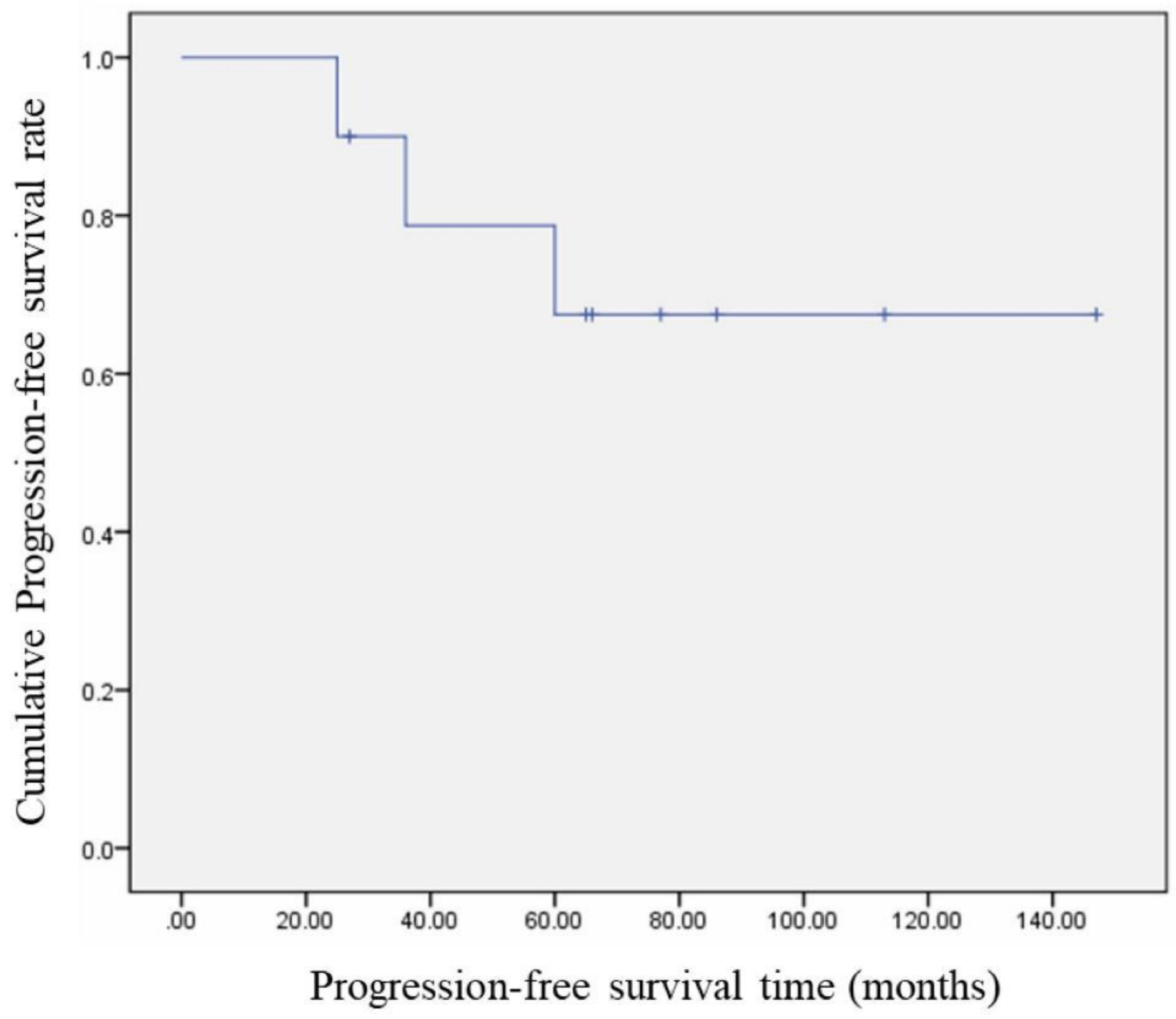

Figure 1

Disease-free survival (DFS). The 5-year and 10-year DFS rates for the entire cohort were both $70 \%$. 